\title{
An interventional multispectral photoacoustic imaging platform for the guidance of minimally invasive procedures
}

\author{
Wenfeng Xia ${ }^{1}$, Daniil I. Nikitichev ${ }^{1}$, Jean Martial Mari ${ }^{1}{ }^{1}$, , Simeon J. West ${ }^{3}$, Sebastien \\ Ourselin $^{1}$, Paul C. Beard ${ }^{1}$ and Adrien E. Desjardins ${ }^{1}$ \\ ${ }^{1}$ Department of Medical Physics and Biomedical Engineering, University College London, \\ Gower street, London WC1E 6BT, United Kingdom; \\ ${ }^{2} \mathrm{GePaSud}$, University of French Polynesia, Faa'a, French Polynesia; \\ ${ }^{3}$ Main Theatres, Maple Bridge Link Corridor, Podium 3, University College Hospital, 235 \\ Euston Rd, London NW1 2BU, United Kingdom;
}

\begin{abstract}
Precise and efficient guidance of medical devices is of paramount importance for many minimally invasive procedures. These procedures include fetal interventions, tumor biopsies and treatments, central venous catheterisations and peripheral nerve blocks. Ultrasound imaging is commonly used for guidance, but it often provides insufficient contrast with which to identify soft tissue structures such as vessels, tumors, and nerves. In this study, a hybrid interventional imaging system that combines ultrasound imaging and multispectral photoacoustic imaging for guiding minimally invasive procedures was developed and characterized. The system provides both structural information from ultrasound imaging and molecular information from multispectral photoacoustic imaging. It uses a commercial linear-array ultrasound imaging probe as the ultrasound receiver, with a multimode optical fiber embedded in a needle to deliver pulsed excitation light to tissue. Co-registration of ultrasound and photoacoustic images is achieved with the use of the same ultrasound receiver for both modalities. Using tissue ex vivo, the system successfully discriminated deep-located fat tissue from the surrounding muscle tissue. The measured photoacoustic spectrum of the fat tissue had good agreement with the lipid spectrum in literature.
\end{abstract}

KEYWORDS: fiber optic; photoacoustic imaging; ultrasound imaging; ultrasonic tracking; minimally invasive procedures

\section{INTRODUCTION}

Medical device guidance is of critical importance for many interventional procedures to identify tissue targets with high sensitivity and specificity, and subsequently to avoid complications. These procedures include fetal interventions, biopsies, central venous catheterisations, tumor ablations, and nerve blocks. ${ }^{1,2}$ Ultrasound (US) imaging is widely used for device guidance in minimally invasive procedures due to its large imaging depth, high spatial resolution and wide availability. However, this modality suffers from limited soft tissue contrast, which can result in insufficient sensitivity and specificity for particular targets.

Optical imaging offers rich optical absorption contrast and specificity, but imaging targets beyond a few $\mathrm{mm}$ depths is challenging due to strong light scattering and absorption in biological tissues. Photoacoustic (PA) imaging is intrinsically a hybrid imaging modality. It takes the advantages of both optical imaging and ultrasound imaging, ${ }^{3}$ could be well suited to guiding certain minimally invasive procedures. It differs from US imaging in terms of how ultrasound is generated. With US imaging, generation is performed by transducers; with PA imaging, generation is performed inside tissues by excitation light pulses. In the latter case, absorption of pulsed light by specific chromophores, followed by rapid and localized temperature rises, and subsequent US generation by thermo-elastic expansion. ${ }^{4}$ The attenuation and distortions of US waves are typically significantly less than those of light waves, optical absorption of tissues can be imaged at large depths. ${ }^{5}$ As PA and US images

Address all correspondence to: Wenfeng Xia, Department of Medical Physics and Biomedical Engineering, University College London, Malet Place Engineering Building, London WC1E 6BT, United Kingdom; Tel: +44 20 76790300; E-mail: wenfeng.xia@ucl.ac.uk.

Opto-Acoustic Methods and Applications in Biophotonics II, edited by Vasilis Ntziachristos, Roger Zemp, Proc. of SPIE Vol. 9539, 95390D · (c) 2015 SPIE · CCC code: 1605-7422/15/\$18 · doi: 10.1117/12.2183798 
can be acquired with the same ultrasound receiver elements, they can be inherently co-registered. A hybrid USPA system can therefore provide both structural information from US imaging and molecular contrast from PA imaging. ${ }^{6}$

There is an increasing interest in developing photoacoustic imaging systems for minimally invasive procedures. ${ }^{7-12}$ Kim et al. ${ }^{7}$ reported a PA imaging system that was based on a commercial US system for guiding sentinel lymph nodes biopsies. In their system, excitation light delivery was provided by a bifurcated optical fiber bundle that was integrated with the US probe. Thus both light illumination and ultrasound detection are located at the tissue surface. From the standpoint of achieving high imaging depths, excitation light delivery from the tissue surface has the disadvantage that light fluence decrease rapidly with depth in tissue due to optical scattering and absorption. To overcome this disadvantage, Piras et al. ${ }^{9}$ developed a system named the photoacoustic needle, in which excitation light was delivered directly to the regions of interest through an optical fiber embedded in a breast biopsy needle. This system provided good sensitivity for identifying targets in phantoms with PA imaging, but with the use of a single light wavelength $(1064 \mathrm{~nm})$, the specificity was limited.

To make use of the spectroscopic optical absorption contrast, we introduce an interventional PA imaging system with multiple excitation light wavelengths to guide minimally invasive procedures. Light was delivered through a spinal needle, and US detection was performed with a commercial US linear array imaging probe. Coregistered photoacoustic and ultrasound images were acquired in an interleaved mode to provide both molecular and structural information of biological tissues. The feasibility of multispectral photoacoustic imaging was demonstrated using an ex vivo tissue sample.

\section{MATERIALS AND METHODS}

\subsection{The imaging system}

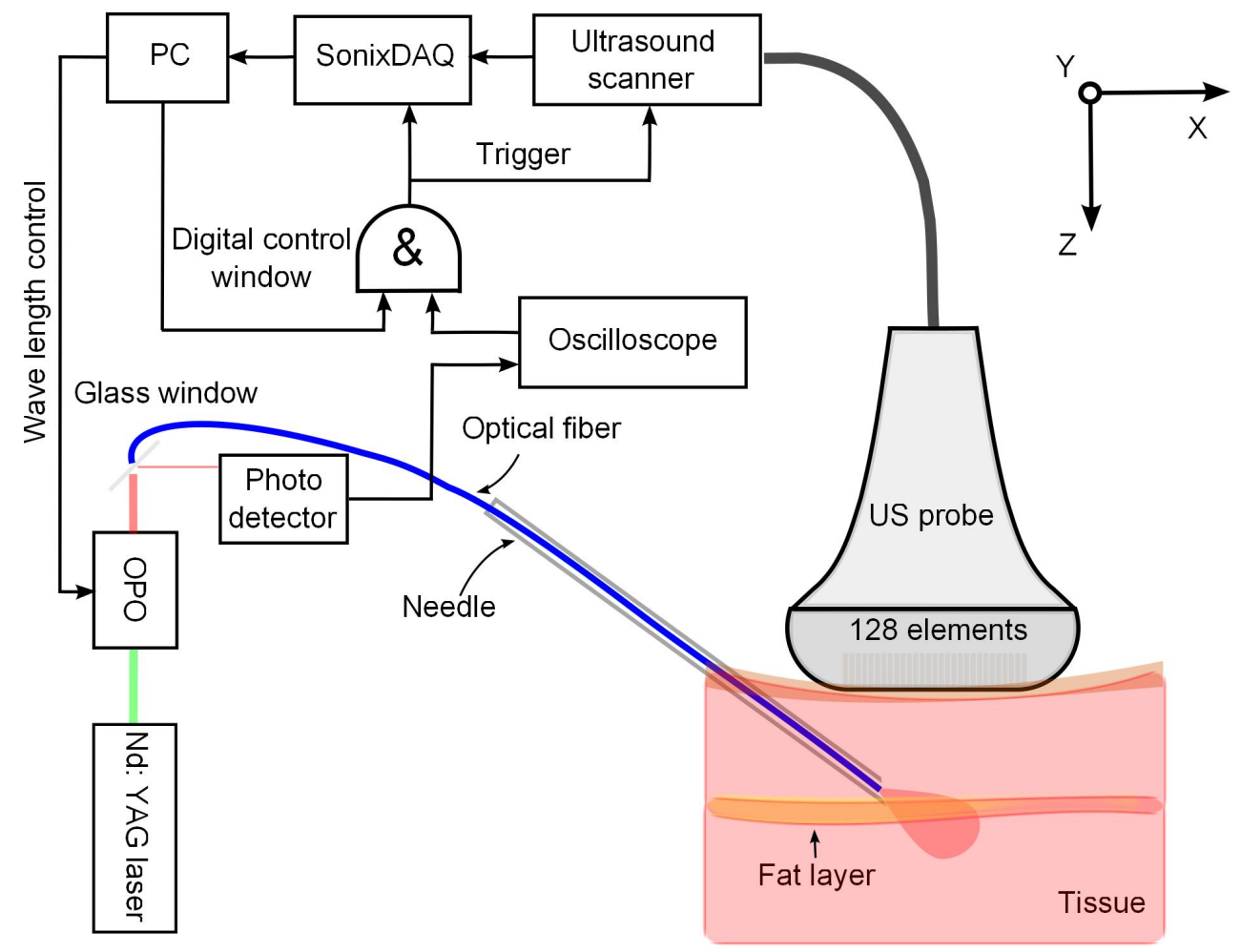

Figure 1: Schematic of the multispectral photoacoustic imaging system.

An optical parametric oscillator (OPO) system (VersaScan L-532, Spectra-Physics, Santa Clara, CA) pumped by a Nd:YAG laser (pulse width $6 \mathrm{~ns}$, repetition rate $10 \mathrm{~Hz}$, Quanta-Ray, INDI-40-10, Spectra-Physics, Santa 
Clara, CA) was used as the excitation light source (Figure 1). The OPO delivers light pulses with two wavelength ranges: the signal $(700-900 \mathrm{~nm})$ and the idler $(1100-2200 \mathrm{~nm})$. Wavelength tuning was controlled by a custom Labview program (National Instruments, Berkshire, UK) using a motorized crystal. The OPO outputs were focused onto separate silica-silica optical fibers. These optical fibers were positioned within the cannula of a 14 gauge spinal needle to deliver light in front of the needle tip, as shown in Figure 1.

US detection used a commercial US imaging system (SonixMDP, Analogic Ultrasound, Richmond, BC, Canada) that was operated in its research mode. The US receiver was a linear array transducer (L14-5/38, Analogic Ultrasound, Richmond, BC, Canada). The transducer array was specified to have a nominal center frequency of $10 \mathrm{MHz}$ with a $-6 \mathrm{~dB}$ bandwidth of $9 \mathrm{MHz}$. Pre-beamformed channel data from the transducer were sampled at $40 \mathrm{MHz}$ with 12-bit ADC by a 128-channel data acquisition system (SonixDAQ, Analogic Ultrasound, Richmond, BC, Canada). Acquisition of PA and US data were triggered by optical pulses. As the OPO operating continuously at $10 \mathrm{~Hz}$, synchronization of wavelength tuning and optical triggering was realized using a digital control window in the Labview program in combination with a logic AND gate. The AND gate has two inputs: the optical trigger pulse and the digital control window (Figure 1). Immediately after an acquisition of RF data for PA imaging, reconstruction was performed using a custom delay-and-sum beam-forming algorithm. The B-mode US images were reconstructed using electronic beam-forming, and the post-beamformed data were displayed alongside the PA images. Offline PA image reconstruction were performed using a Fourier-domain reconstruction algorithm, ${ }^{13}$ which was implemented with the k-Wave MATLAB toolbox. ${ }^{14}$

\subsection{Ex vivo tissue imaging}

To obtain a preliminary indication of the system's potential to identify tissue targets such as nerves, ex vivo fat tissue samples were used as absorbing targets, in which lipid absorption is dominant. ${ }^{15,16}$ Multispectral photoacoustic imaging was performed on ex vivo porcine tissues comprising a fat layer sandwiched by two muscle layers. The needle was inserted toward the fat layer at an angle of around 20 degrees (relative to the surface normal). Photoacoustic imaging at wavelengths from 1160 to $1300 \mathrm{~nm}$ was performed with a distance between the needle tip and the fat layer of approximately $5 \mathrm{~mm}$. Average PA image amplitudes were calculated over a 2 $\mathrm{mm} \times 2 \mathrm{~mm}$ region of interest (ROI) in front of the fiber tip, within the fat layer.

\section{RESULTS}

With ex vivo tissue sample experiments, PA and US images had excellent spatial correspondence [Figure 2(a)(d)]. A high intensity region was apparent at the needle tip location in the PA images [Figure 2(a)-(b)]. At an excitation wavelength of $760 \mathrm{~nm}$, no signal was apparent in the fat layer. At an excitation wavelength of 1210 $\mathrm{nm}$, which lipid absorption spectrum has a local maximum, a strong signal was clearly visible in the ROI, which was attributed to be generated from the fat layer [Figure 2(d)]. The PA spectrum obtained as an average across the ROI was found to be very similar to the optical absorption spectrum of lipid [Figure 2(e)].

\section{DISCUSSION AND CONCLUSIONS}

An interventional multispectral photoacoustic imaging system was introduced. It delivers excitation light directly to targets through an optical fiber embedded into a medical needle. Tissue target at depths greater than 20 $\mathrm{mm}$ could be clearly observed. In this way, the system has strong potential to overcome the imaging depth limitations that arise with surface illumination. Using multiple wavelengths, the system provides image contrast for different chromophores such as lipid and hemoglobin that could be used to differentiate procedure targets, which in turn could result in safer and more accurate minimally invasive procedures.

\section{ACKNOWLEDGMENTS}

This work was supported by an Innovative Engineering for Health award by the Wellcome Trust [WT101957] and the Engineering and Physical Sciences Research Council (EPSRC) [NS/A000027/1], by a Starting Grant from the European Research Council [ERC-2012-StG, Proposal 310970 MOPHIM], and by an EPSRC First Grant [EP/J010952/1]. 

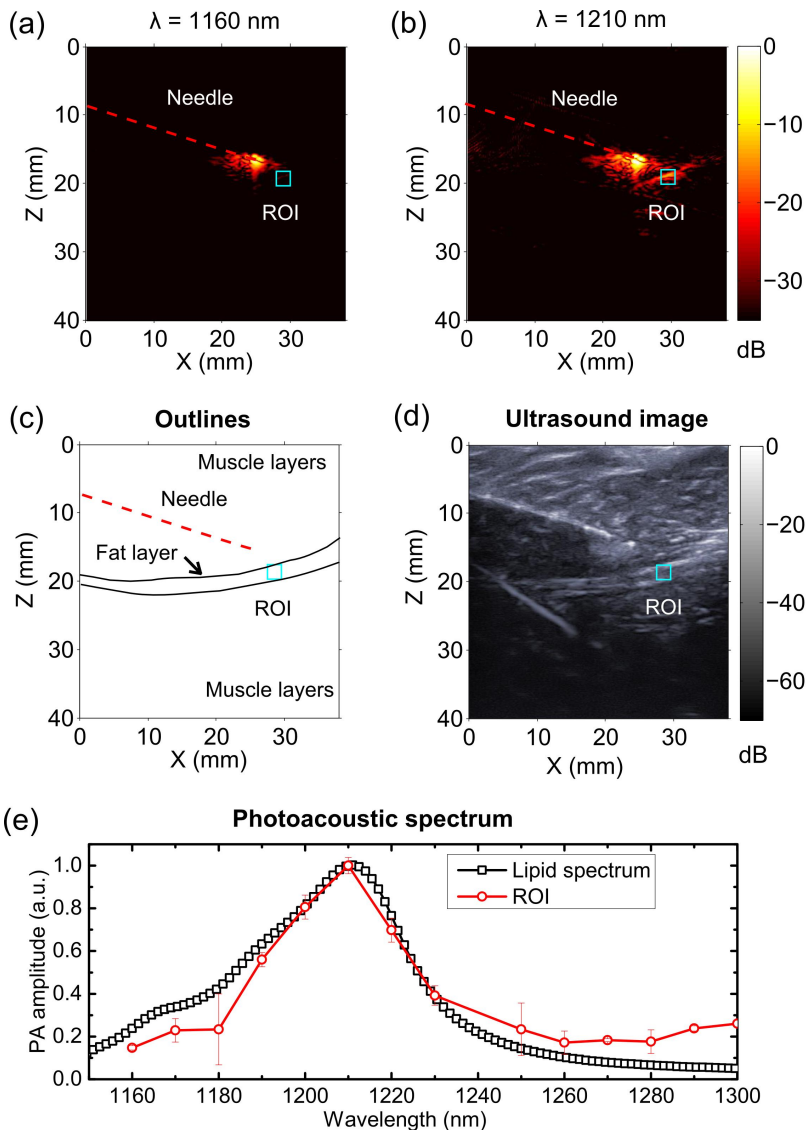

Figure 2: Multispectral photoacoustic imaging of a porcine tissue sample ex vivo. The sample consisted of a fat layer which was sandwiched by two muscle layers. Photoacoustic images at excitation light wavelength of $1160 \mathrm{~nm}$ and $1210 \mathrm{~nm}$ are shown in (a) and (b). Co-registered ultrasound image and the image outlines are shown in (d) and (c), respectively. The photoacoustic spectrum obtained from the region of interest (ROI) indicated in (A) and (B) is compared with the optical absorption spectrum of lipids in (e). The values represent average values from 15 image frames and the error bars represent standard deviations.

\section{REFERENCES}

1. M. Bluvol, A. Shaikh, A. Kornecki, D. D. R. Fernandez, D. Downey, A. Fenster, "A needle guidance system for biopsy and therapy using two-dimensional ultrasound," Med. Phys. 35, 617-628 (2008).

2. A. G. Randolph, D. J. Cook, C. Gonzales, A. Calle, C. G. Pribble, "Ultrasound guidance for placement of central venous catheters: A meta-analysis of the literature," Critical Care Medicine 24(12), 2053-2058 (1996).

3. L. V. Wang, S. Hu, "Photoacoustic tomography: in vivo imaging from organelles to organs," Science 335(6075), 1458-1462 (2012).

4. P. C. Beard, "Biomedical photocoustic imaging," Interface Focus 1(4), 602-631 (2011).

5. W. Xia, W. Steenbergen, and S. Manohar, "Photoacoustic mammography: prospects and promises," Breast Cancer Management 3(5), 387-390 (2014).

6. T. J. Allen, A. Hall, A. P. Dhillon, J. S. Owen, P. C. Beard, "Spectroscopic photoacoustic imaging of lipid-rich plaques in the human aorta in the 740 to $1400 \mathrm{~nm}$ wavelength range," J. Biomed. Opt. 17(6), 061209 (2012).

7. C. Kim, T. N. Erpelding, K. Maslov, L. Jankovic, W. J. Akers, L. Song, S. Achilefu, J. A. Margenthaler, M. D. Pashley, and L. V. Wang, "Handheld array-based photoacoustic probe for guiding needle biopsy of sentinel lymph nodes," J. Biomed. Opt. 15(4), 046010 (2010).

8. J. Su, A. Karpiouk, B. Wang and S. Emelianov, "Photoacoustic imaging of clinical metal needles in tissue," J. Biomed. Opt. 15(2) 021309, (2010) 
9. D. Piras, C. Grijsen, P. Schütte, W. Steenbergen, and S. Manohar, "Photoacoustic needle: minimally invasive guidance to biopsy," J. Biomed. Opt. 18(7), 070502 (2013).

10. M. A. Lediju Bell, N. P. Guo, D. Y. Song, J. U. Kang, and E. M. Boctor, "in vivo visualization of prostate brachytherapy seeds with photoacoustic imaging," J. Biomed. Opt. 19(12), 126011 (2014).

11. M. A. Lediju Bell, X. Guo, D. Y. Song, and E. M. Boctor, "Transurethral light delivery for prostate photoacoustic imaging," J. Biomed. Opt. 20(3), 036002 (2015).

12. L. Lin, J. Xia, T. T. W. Wong, L. Li, and L. V. Wang, "in vivo deep brain imaging of rats using oral-cavity illuminated photoacoustic computed tomography," J. Biomed. Opt. 20(1), 016019 (2015).

13. K. P. Köstli, and P. C. Beard, "Two-dimensional photoacoustic imaging by use of Fourier-transform image reconstruction and a detector with an anisotropic response," Appl. Opt. 42(10), 1899-1908 (2003).

14. B. E. Treeby, and B. T. Cox, "k-Wave: MATLAB toolbox for the simulation and reconstruction of photoacoustic wave fields," J. Biomed. Opt. 15(2), 021314 (2010).

15. T. P. Matthews, C. Zhang, D. Yao, K. Maslov, L. V. Wang, "Label-free photoacoustic microscopy of peripheral nerves," J. Biomed. Opt. 19(1), 016004 (2014).

16. J. M. Mari, S. J. West, P. C. Beard, A. E. Desjardins, "Multispectral photoacoustic imaging of nerves with a clinical ultrasound system," Proc. SPIE 8943, 89430-W1 (2014). 